\title{
A Modified Watershed Algorithm for Stellar Image
}

\author{
Dibyendu Ghoshal \\ Associate Professor, Department of ECE \\ National Institute of Technology \\ Agartala, Tripura, Pin - 799001
}

\author{
Pinaki Pratim Acharjya \\ Assistant Professor, Department of CSE \\ Bengal Institute of Technology and Management \\ Santiniketan, West Bengal, Pin - 731236
}

\begin{abstract}
A modified gray scale watershed image segmentation algorithm suitable for low contrast image has been proposed. Digital images acquired from far away stellar objects (like stars, planets, galaxies, comets etc.) are prone to be severally affected by various types of noises and the contrast of these categories of images are generally found to be low. In present study, a preserving de noising method is presented by a contrast adjustment based on adaptive histogram equalization technique. The proposed method has been found to yield satisfactory segmentation of the stellar images. The entropy of the original and the segmented image is compared and the result confirms to the reality.
\end{abstract}

\section{Keywords}

Stellar Image, Segmentation, Watersheds.

\section{INTRODUCTION}

Image segmentation has been an essential step in digital image processed for most subsequent image analysis and image understanding activities. In specific, many of the prevailing method for image description and recognition [12], image visualization [3-4], and object based image compression [5-7], highly depend on the segmentation result obtained from previous stage. The segmentation problem [10], [12] involves separating or partitioning of an image into a number of homogenous and spatially connected group of pixels called super pixels, so it has addition of two neighborhood segments produces a heterogeneous segment. As an alternative approach, segmentation can be thought of as a pixel labeling cum mathematical morphological process in the same that all the pixels belonging to the same homogeneous region are assigned the same label and theme and several techniques to define homogeneity of a region based on a particular objective of image segmentation process.

Stellar images of various planets, stars, galaxies, comets, nebula and other various extra terrestrial moving objects have occupied a significant place in radio astronomy, space research and preparation of distance space vehicle movements (manual or robotized) [8]. The images of stellar objects have been acquired either through satellite imagery or Hubble space telescope. These images are very much prone to be affected by a verity of noise like Gaussian noise, Rayleigh noise, Impulse noise and Speckle noise. At the same time, the contrast of these images have been generally found to be low to very long distance and atmosphere turbulence and disturbance and variation of temperature during the transmission of them through space media. The segmentation of low contrast images have always posed problem due to processing problem arising from inability to directly apply the watershed algorithm on the image. Rather it has been found to be more suitable to apply watershed to the gradient of the image instead of the original image [20]. In this approach, the original minima of catchment basins correlate with the small values of the image gradient value. Thus the study of the effect of application of varies modified version or combination of morphological based watershed algorithm [9], [14-19] on low contrast images of stellar objects have become worthwhile and interesting.

A good number of methods have been proposed and applied to solve the problem related to digital image segmentation [11], [13]. These techniques can be broadly classified into the following categories as histogram based techniques, edge based techniques, region based techniques, markov random field based techniques and hybrid techniques. All the above techniques have got certain advantages as well as some complexity compared to the others, the present technique would fall within the group of processing having adaptive histogram equalization as a preprocessing step for low contrast stellar images.

\section{THE WATERSHED: A TOOL FOR MORPHOLOGICAL SEGMENTATION}

Watershed algorithm is based on morphological process although it can be mixed up with edge based segmentation to yield a hybrid technique. In mathematical morphology, an image may be considered as a topographical surface. This is accomplished (the image intensity) as an altitude. The image pixels having same intensity value constitute one segment within the region of the image. The set of pixels along which the gray levels changes sharply gives rise to an edge. Normally, images acquired by various techniques in the electromagnetic spectrum, possesses a large no of discontinuities in the intensity and these ultimately give rise to over segmentation when morphological segmentations like watersheds are carried out. Thus to avoid over segmentation phenomena, a thorough preprocessing is necessary. A method which is commonly used to control over segmentation is based on the concept of marker. The markers are connected components of an image. There can be two types of markers, internal and external. Internal markers are associated with object of interest and external markers are associated with the back ground of the original image.

\section{BASIC PHILOSOPHY BEHIND THE WATERSHED ALGORITHM}

In general segmentation problem indicates partitioning the image according any predefined criterion. A gradient image may be considered as a topological surface where the numerical value of each pixel indicates the evaluation of their points. Smooth surface on the image plane can be decomposed into crests and troughs after a study of the critical points and slope lines of a surface. The watershed algorithm may be simulated in two ways with a common objective of viewing the elevation in the gray scale. The methods are either defined in term of the drainage pattern of rain fall. The zones which drawn to the same points are defined as a part of the same watershed. The same approach can be applied to image by viewing intensity as heights where the gradient is utilized to predict the direction of drainage for each point in the image. One set of points that drain to every local intensity 
minimum that can be identified. These connected or disjoint regions are termed as the watershed as the watershed region or catchment basins of the image. The gray level profile of an image data and corresponding watershed segmentation local minima and maxima are shown in figure 1 and figure 2 .

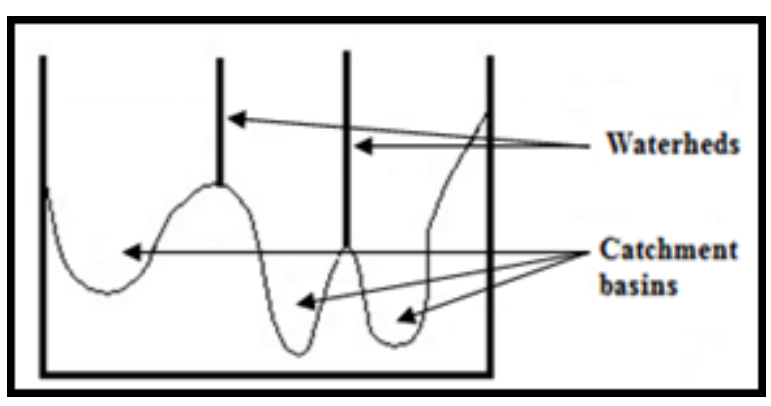

Fig 1: Watershed segmentation-local minima of gray level yield catchment basins. Local maxima define the watershed lines.

The second technique is also related to geographical approach and using this analogy, one vessel can be pierced at the bottom and the pierced holes would acts as a regional minimum. When this vessel (i.e., the image) would be submerged into a large water body, water would enter the vessel and the water level would rise gradually. It would flood the basin (i.e. the vessel) corresponding to various movement. The dams are created to avoid the merging of different water originating from different minima (i.e. holes). The dams ultimately come out to be watershed lines where the surface is fully immersed. In one dimensional view point, the regional maxima of the function turn out to be watershed line. In two dimension (which is real case for a two dimensional image plane), the place where two basins meet for the first time is a saddle point in the image. The set of cross lines of the image emerging from the saddle pints can be considered as watershed lines.

\section{MATHEMATICAL FORMULATION RELATED TO WATERSHED}

The watershed method in image processing as shown in figure 2 , summarized by suppose the lower point in the image are $\mathrm{B}_{1}, \mathrm{~B}_{2} \ldots \mathrm{B}_{\mathrm{Z}}$ to be coordinate of these points for the image I $(\mathrm{i}, \mathrm{j})$ and $\mathrm{CB}_{\mathrm{m}}$ refers to the points of catchment basins associated with minimum region $\mathrm{B}_{\mathrm{Z}}(\mathrm{x}, \mathrm{y})$ represented by $\mathrm{X}$ [n] accordingly $\mathrm{I}(\mathrm{x}, \mathrm{y})<\mathrm{n}$.

$$
X[n]=\{(x, y)\} \mid I(x, y)<n\}
$$

$\mathrm{X}[\mathrm{n}]$ is the coordinate of points in $\mathrm{I}(\mathrm{i}, \mathrm{j})$ geometrically lying under the plain $I(i . j)=n$. Topographically the image filled with water in integer filling increments begin from $n=t_{\max }-1$ to $\mathrm{n}=\mathrm{t}_{\min }+1$.

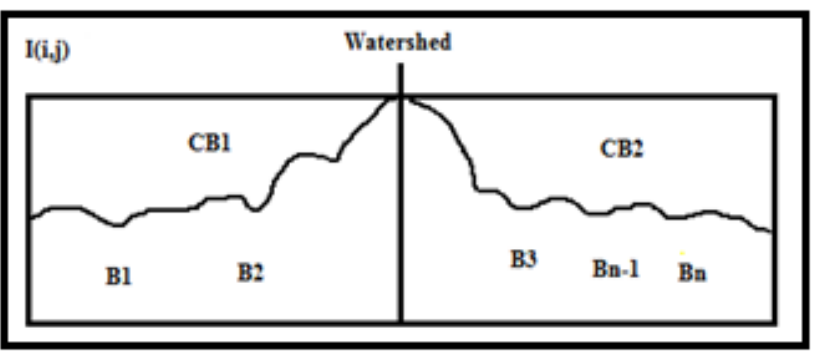

Fig 2: The watershed method.
The number of points under the fluid is necessary, due to that marker will used in black color for the coordinates in $X[n]$ which are below the level $I(i, j)=n$ and the other point in white color.

$$
C_{n} B_{m}=C B_{m} \cap X[n]
$$

Where $C_{\mathrm{m}} B_{\mathrm{m}}$ represents the coordinates in catchment basins related to $B_{m}$, which are fluid filling at the level $\mathrm{n}$. Then let $\mathrm{C}[\mathrm{n}]$ refer to the union of the filling fluid of the points of catchment basins of level $\mathrm{n}$ :

$$
C[n]=\bigcup_{m=1}^{z} C_{n} B_{m}
$$

Finally $\mathrm{C}_{\mathrm{tmax}}+1$ refer to all catchment basins union.

$$
C_{t_{\max +1}}=\bigcup_{m=1}^{Z} C B_{m}
$$

From equation 2 and $3, \mathrm{C}[\mathrm{n}]$ is a subgroup of $\mathrm{X}[\mathrm{n}]$ accordingly; then watershed lines is prepared when $\mathrm{C}$ $\left[t_{\min }+1\right]=\mathrm{X}\left[\mathrm{t}_{\min +1}\right]$. Then the procedure follows to reconstruct $\mathrm{C}[\mathrm{n}-1]$ at level n.C[n] can be obtained from $\mathrm{C}[\mathrm{n}$ 1] by assuming $\mathrm{S}$ as the set of the connected component in $\mathrm{X}[\mathrm{n}]$, at $\mathrm{s} \in \mathrm{S}[\mathrm{n}]$ there are three assumption i) $\mathbf{s} \cap \mathrm{C}[\mathrm{n}-1]$ is empty .ii) $\mathbf{s} \cap \mathrm{C}[\mathrm{n}-1]$, contains more than one connected component of $\mathrm{C}[\mathrm{n}-1]$ and this lead to $\mathrm{s}$ is incorporated in to $\mathrm{C}$ [n-1] to produce $C[n]$. iii) In last case $\mathbf{s} \cap \mathrm{C}[\mathrm{n}-1]$ contains more than one connected component of $\mathrm{C}$ [n-1].

\section{PROPOSED ALGORITHM}

The image (Fig 3), being a distance object has low contrast. These may be various interferential factors in such type of images such as non uniform illumination, variation in temperature of the ambience, atmospheric disturbance and turbulence, variation in the intensity and contrast etc. All these factors would enhance the difficulty of segmentation of galactic images. Thus a meaningful and effective measure is warranted to alleviate the adverse influence. So some image processing method would have to be applied and the image preprocessing stage comprises a number of methods such as gray level transformation, contrast enhancement, image de noising, image smoothing, sharpening and so on.

This approach deals with catchment basins and watershed ridge lines in an image by assuming it as a surface where light pixels are low. But most important contrast of an image is very important for image segmentation. It is the difference in visual properties that makes difference between one object of an image from the background or we can say it is the difference in the color and brightness of the object. So for satisfactory image segmentation image with satisfactory resolution is essential. In this approach, for low contrast stellar images we have used watershed with markers. In the initial step shown in figure 4, one stellar image is chosen, where contrast of that image is low and appropriate area is cropped which is shown in figure 5. We need to adjust the contrast. Contrast adjustment is necessary for good image understanding and analyzing. In figure 6 , the image contrast is adjusted with adaptive histogram equalization method. In figure 7 we have applied threshold. In figure 8 , we have clean up and overlay the perimeter on the original image by using imoverlay function, This function takes input image and a binary mask and generates an output image, where masked pixels have been replaced by a specified color. In figure 9, we apply markers for object and background. The extended maxima operator is used to identify groups of pixels that are 
significantly higher than their immediate surroundings. Again cleaning up and overlying is there in figure 10 and compute the complement of the image so that the peaks become valleys and modifies the image using imimposemin function. Finally we will sum up the watershed transform in figure 11 along with consequence examination. In this paper we study this approach with a low contrast image of a galaxy and detecting it. The flowchart of the proposed method is given below in figure 4.

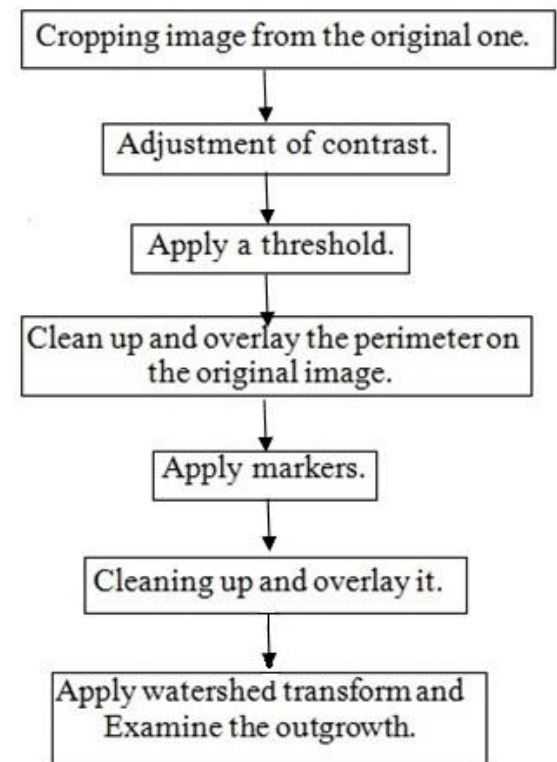

Fig 3: Flowchart of the proposed method.

\section{IMPLIMENTATION RESULTS}

The proposed algorithm has been applied on the image of a galaxy. Figure 3 to 8 shows the step wise output image which have been obtained by applying each process step of the algorithm one often another. Galaxy, being a stellar object, is very much prone to be effected by various types of random noises which include Gaussian, Salt and pepper, Rayleigh, Gamma and Exponential noise. Following figures (Figure 5 to 11) will give us the clear idea about the step wise results of the proposed algorithm.

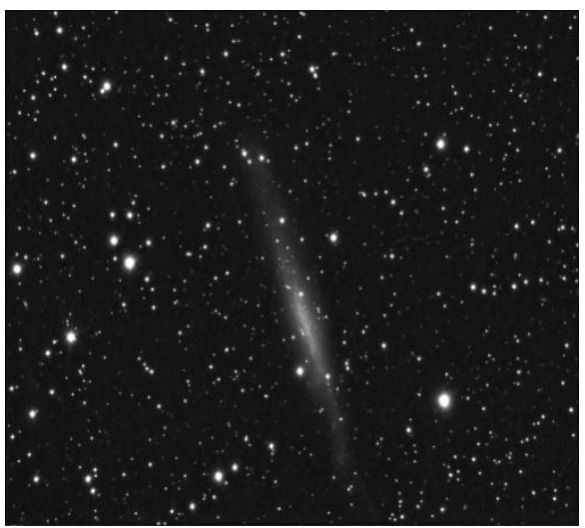

Fig 4: Original image.

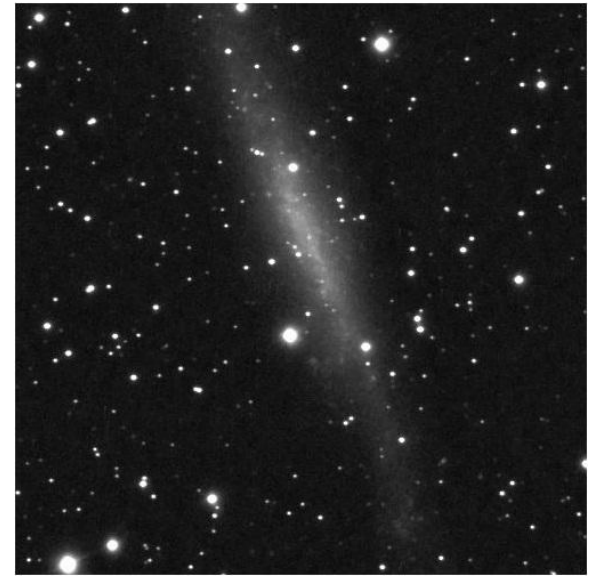

Fig 5: Cropped image from the original one.

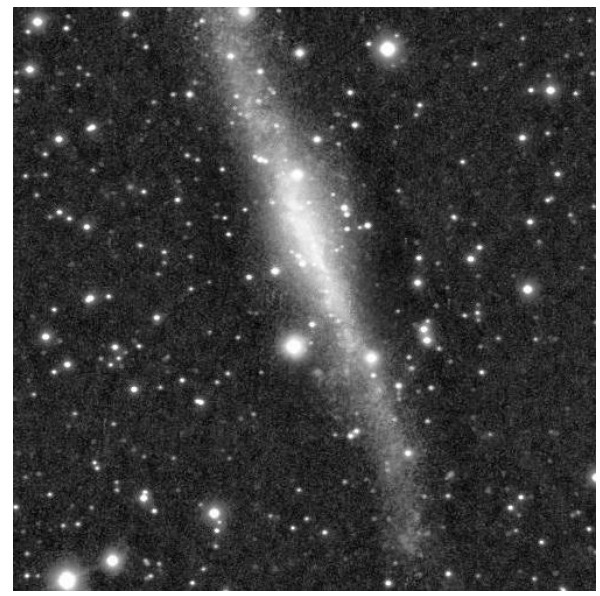

Fig 6: Image with contrast adjustment.

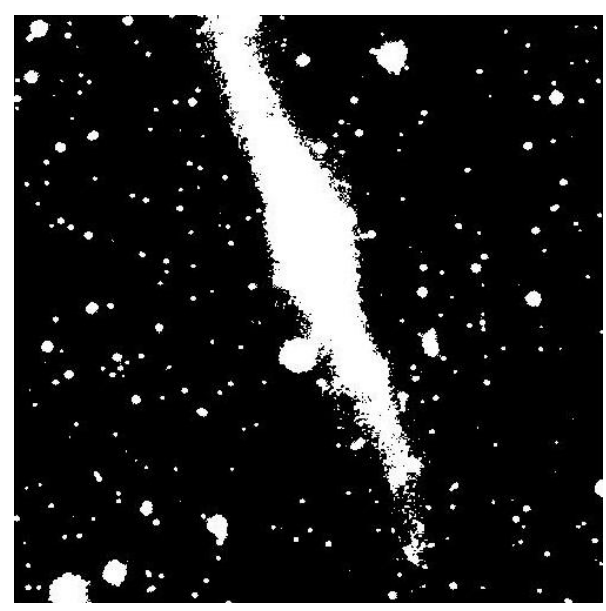

Fig 7: Image by applying a threshold. 


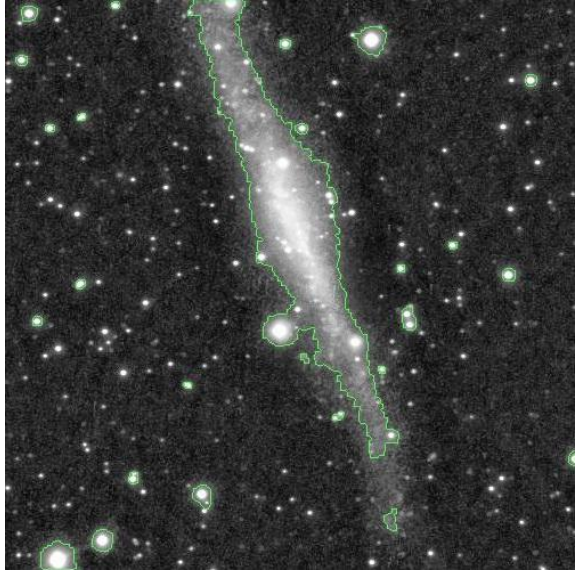

Fig 8: cleaning up and overlaying the perimeter on the original image.

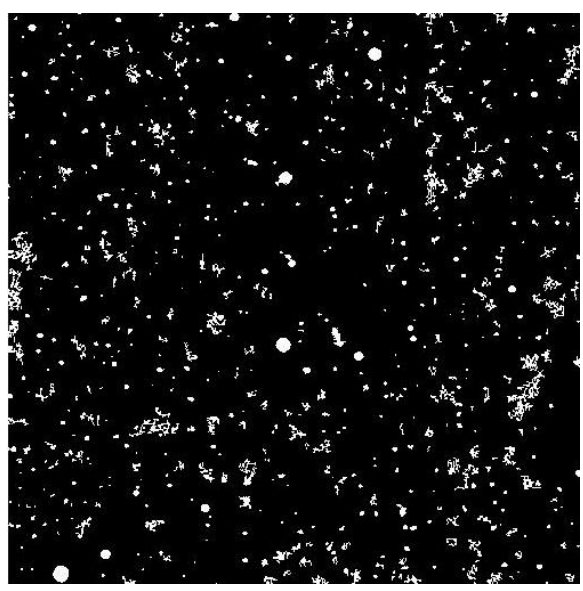

Figure 9: Image by applying markers.

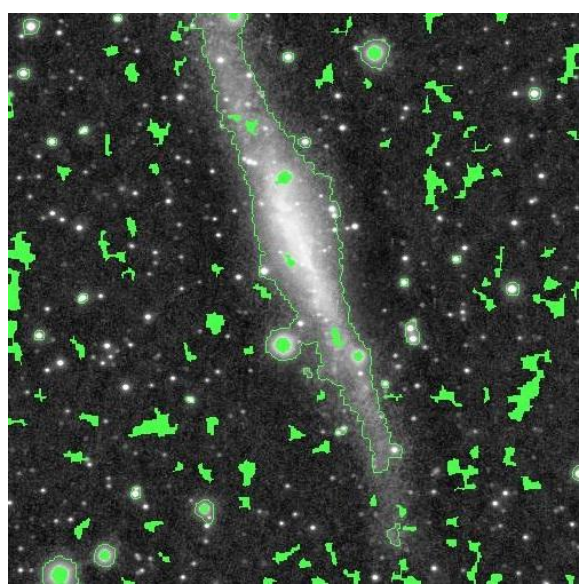

Fig 10: cleaning up and then overlay it.

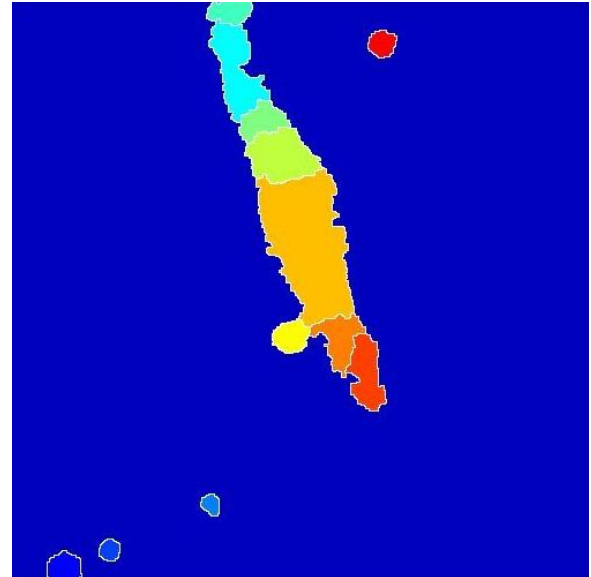

Fig 11: Final image by applying watershed transform.

The image of stellar objects, in addition to be infected by noise, generally possesses low intensity and low contrast owing to the very long distance of these galactic images which are generally acquired from satellites or through space telescopes. In below The present study has tried to overcome the problem of the low contrast image and at the same time it has tried to avoid over segmentation. The performance of Segmentation algorithm is measured with the help of Entropy and (see Table 1) as in term of visual quality of the original image and the resulted image.

\section{Table I- statistical measurement}

\begin{tabular}{|c|c|}
\hline IMAGE & ENTROPY \\
\hline Original Image (Fig 4) & 4.6265 \\
\hline Segmented Image (Fig 11) & 3.4810 \\
\hline
\end{tabular}

The image entropy can provide a good level of information to describe a given image. Low entropy images, such as those containing a lot of black sky, have very little contrast and large runs of pixels with the same values. An image that is perfectly flat will have entropy of zero. Consequently, they can be compressed to a relatively small size. On the other hand, high entropy images such as an image of heavily cratered areas on the moon have a great deal of contrast from one pixel to the next and consequently cannot be compressed as much as low entropy images.

\section{CONCLUTSONS}

The present study has aimed at to provide an easy method of the segmentation of the segmentation of stellar objects based on mathematical morphology. The stellar objects like galaxy, nebulae, stars, comets are situated at a very long distance from the earth. Hence their images are acquired either via satellite or through space telescope. Both the means yield low intensity, low contrast image. Results obtained from the application of watershed algorithm over the gradient image are not always found to be satisfactory. The present method has been found to yield better output (segmented image) in term of image quality, clarity and contrast with the avoidance of over segmentation.

\section{ACKNOWLEDGEMENT}

The authors acknowledge the constant inspiration and encouragement from Professor P. K. Bose, Director, National Institute of Technology, Agartala. 


\section{DEDICATION}

One of the others (Dibeyendu Ghoshal) dedicates the entire study to the loveliest and loving memory of his only one and younger sister Kumari Sumita Ghoshal who herself was a gem of the scholars, a symbol of wisdom and art, peerless beauty and simplicity, unfathomable knowledge and generosity.

\section{REFERENCES}

[1] P. Suetnes, P.Fua and A. J. Hanson, "Computational strategies for object recognition," ACM Computing Surveys, Vol. 24, pp. 05-61, 1992.

[2] R. Besl, R. Jain, "Three dimensional object recognition," ACM Computing Surveys, Vol. 17, pp. 75-145, 1985.

[3] K. Hohne, H. Fuchs, S. Pizer, "3D imaging in medicine: Algorithms, systems, Applications", Berlin, Germany, Springer -Verlag, 1990.

[4] M. Bomans, K. Hohne, U. Tiede and M. Riemer, "3D segmentation of MR images of the head for 3D display," IEEE Transactions on Medical imaging, Vol.9, pp. 253277, 1990.

[5] M. Kunt, M. Bernard and R. Leonardi, "Recent results in high compression image coding," IEEE Trans. on Circuits and Systems, Vol.34, pp.1306-1336, 1987.

[6] P. Willemin, T. Reed and M. Kunt, "Image sequence coding by split and merge," IEEE Trans. on Circuits and Systems, Vol.34, pp.1306-1306, 1987.

[7] F. D. Natale, G. Desoli, D. Glusto and G. Vernazza, "Polynomial approximation and vector quantization: A region based integration," IEEE transections on Communications, Vol.43, 1995.

[8] K. Haris, "A hybrid algorithm for the segmentation of 2D and 3D images," Master's thesis, University of Crete, 1994.

[9] R. Harlick and L. Shapiro, "Image segmentation technique," CVGIP, Vol.29, pp. 100-137, 1985.
[10] Vicent L. Solille P, Watershed in digital spaces, "An efficient algorithm based immersion simulations", IEEE Transections PAMI, pp. 538-598, 1991.

[11] Gonzalez \& Woods, Digital Image Processing, 3rd edition, Prentice Hall India, 2008.

[12] K. Haris,"Hybrid image segmentation using watersheds and fast region merging," IEEE Trans Image Processing, 7(12), pp. 1684-1699, 1998

[13] Jos B.T.M. Roerdink and Arnold Meijster.: "The watershed transform: Definitions, algorithms and parallelization strategies," Fundamental Informatics, Vol. 41, pp.187-228, 2001.

[14] Hua LI et al., "An improved image segmentation approach based on level set and mathematical morphology," GREYC-ISMRA, CNRS 6072, 6 Bd Maréchal Juin, 14050 Caen, France.

[15] Mahua Bhattacharya, Arpita Das, "A Study on Seeded Region Based Improved Watershed Transformation for Brain Tumor segmentation," Indian Institute of Information Technology \& Management, Gwalior Morena Link Road, Gwalior-474010.

[16] D. Marr and E. Hildreth, "A theory of edge detection," Proc. R. Soc. London, no 207, pp. 187-217.

[17] Chen Pan, Congxun Zheng, Hao-Jun Wang "Robust Color Image Segmentation Based On Mean Shift And Marker-controlled Watershed Algorithm", Second International Conference on Machine Learning and Cybernetics, Wan, ,pp 2752-2756, 2003.

[18] F. Meyer, S. Beucher, "Morphological Segmentation", Journal of Visual Communication and Image Representation, 1, pp. 21-46, 1990.

[19] N. Pal and S. pal, "A review on image segmentation techniques," Pattern Recognition, Vol.26, pp.1277-1294, 1994.

[20] K. Haris, G. Tziritas and S. Orphanoudakis, "Smoothing 2D or 3D images using local classification," in Proc. EUSIPCO, Edinburg, U.K., 19994. 